\title{
Mechanism of BMP9 promotes growth of osteosarcoma mediated by the Notch signaling pathway
}

\author{
PENG LIU $^{1 *}$, YUQIANG MAN $^{1 *}$, YANQUN WANG $^{2}$ and YUSONG BAO ${ }^{1}$ \\ ${ }^{1}$ Department of Orthopedics, Zaozhuang Municipal Hospital, Zaozhuang, Shandong 277101; \\ ${ }^{2}$ Department of Orthopedics, People's Hospital of Shizhong District, Zaozhuang, Shandong 277102, P.R. China
}

Received October 27, 2015; Accepted December 30, 2015

DOI: 10.3892/ol.2015.4067

\begin{abstract}
Bone morphogenetic protein 9 (BMP9), also known as growth differentiation factor 2, plays a key role in promoting osteosarcoma growth. However, the underlying mechanism remains to be determined. The aim of the present study was to determine the mechanism of BMP9 promoting the growth of osteosarcoma mediated by the Notch signaling pathway. Osteosarcoma cell lines, 143B and MG63, were used for the in vitro experiments. Cell proliferation, cell migration and cell cycle transformation were monitored under various settings. The control and experimental groups used in the present study were BMP9 adenovirus (AdBMP9), a recombinant adenovirus expressing the dominant-negative mutant of Notch1 (AdR-dnNotch1), AdBMP9+AdR-dnNotch1 and AdBMP9+compound E (blocker of the Notch signaling pathway). The results showed that Notch ligands DLL1, JAG1 and JAG2, as well as Notch receptors Notch1, Notch2 and Notch 3 were markedly expressed in the two cell lines. Cell proliferation and migration ability increased in the AdBMP9 group and were higher than that in the AdBMP9+AdR-dn Notch1 and AdBMP9+compound E group. Cell proliferation and migration in the AdR-dnNothc1 group was lower than that in the AdBMP9 group, although the differences were not statistically significant $(\mathrm{P}>0.05)$. The cell cycle ratio in the $S / G 2$ phase increased significantly in the AdBMP9 group and was higher than that in the AdBMP9+AdR-dnNotch1 and AdBMP9+compound E groups. By contrast, the ratio of the cell cycle in S/G2 phase in the AdR-dnNotch1 group was lower than that in the AdBMP9 group. The differences were not statistically
\end{abstract}

Correspondence to: Dr Yusong Bao, Department of Orthopedics, Zaozhuang Municipal Hospital, 41 Longtou Road, Zaozhuang, Shandong 277101, P.R. China

E-mail: ic2200220@163.com

*Contributed equally

Key words: Notch signaling pathway, bone morphogenetic protein 9, osteosarcoma, a recombinant adenovirus expressing the dominant-negative mutant of Notch1 significant $(\mathrm{P}>0.05)$. In conclusion, the results showed that the Notch signaling pathway plays an important role in mediating the growth of osteosarcoma promoted by BMP9.

\section{Introduction}

Bone morphogenetic protein 9 (BMP9) also known as growth differentiation factor 2, plays a key role in the promotion of osteosarcoma growth $(1,2)$. Findings of previous studies suggest that the Notch signaling pathway plays a central role in mediating the signal activity of BMP9 $(3,4)$. The Heyl gene significantly increases during pluripotent stem cell differentiation induced by BMP9 (5). The Heyl gene is highly conserved and the classical target gene of Notch signaling pathway is involved in the regulation of cell proliferation, differentiation and apoptosis (6). Additionally, there is an interaction between the Notch signaling pathway and the TGF- $\beta$ /BMP signaling pathway (7). It has also been reported that the abnormal Notch signaling pathway resulted in the abnormality of cell proliferation and differentiation $(8,9)$.

The Notch signaling pathway is a mechanism whereby adjacent cells communicate with each other, conveying spatial information and genetic instructions for cells (9). The abnormal Notch signaling pathway occurred in various common types of human malignant tumors, such as T-cell leukemia, lung cancer, colon cancer, prostatic cancer, and breast cancer. Based on these findings, this study verified that the mechanism of BMP9 promotes the growth of osteosarcoma mediated by the Notch signaling pathway in vitro.

\section{Materials and methods}

Materials. Osteosarcoma cell lines 143B and MG63 were purchased from the American Type Culture Collection (Manassas, VA, USA) and saved by the Bone and Molecular Oncology Research Center of the University of Chicago (Chicago, IL, USA). The adenovirus vector BMP9 adenovirus (AdBMP9) was constructed and saved by the Bone and Molecular Oncology Research Center of the University of Chicago. Compound E ( $\gamma$-secretase inhibitor XXI) blocker of the Notch signaling pathway was purchased from Enzo Life Sciences, Inc. (Farmingdale, NY, USA), dissolved in DMSO and kept in the freezer at $-80^{\circ} \mathrm{C}$. 


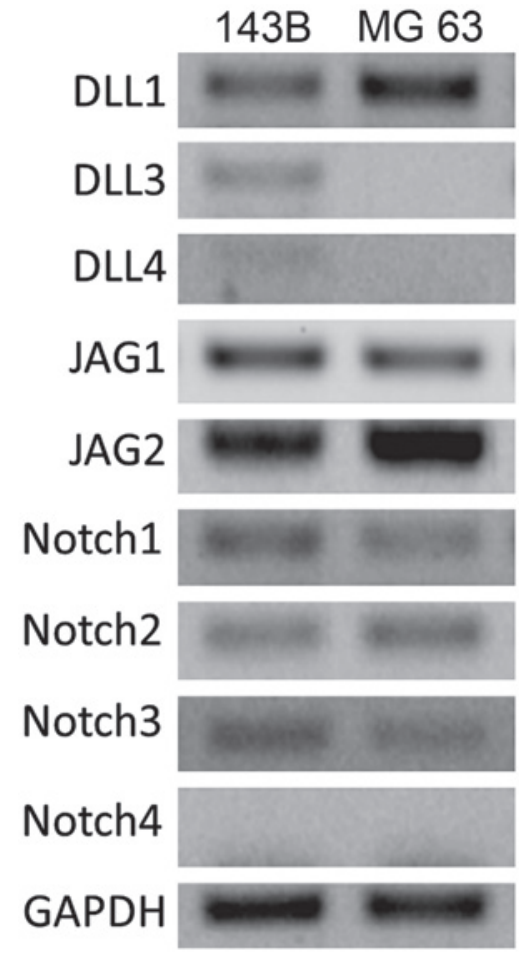

Figure 1. Expression of the ligands and Notch receptors.

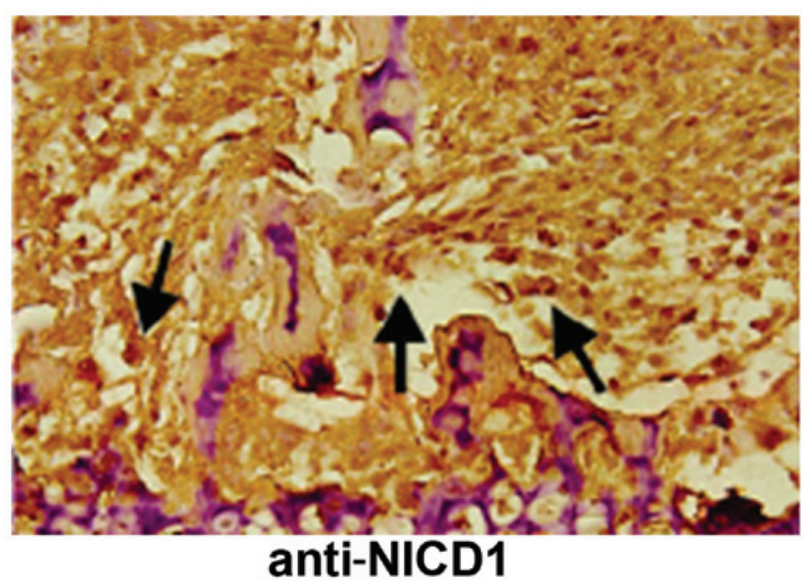

Figure 2. The expression of Notch intracellular domain NICD1.

Experimental grouping. Control and experimental groups used in this study were as follows: i) AdBMP9, ii) a recombinant adenovirus expressing the dominant-negative mutant of Notch1 (AdR-dnNotch1), iii) AdBMP9+AdR-dnNotch1 and iv) AdBMP9+compound $\mathrm{E}$. These were used to process the 143B and MG63 cell lines, and to observe how they affect cell proliferation, migration and transformation of the cell cycle.

Construction of AdR-dnNotch. Polymerase chain reaction (PCR) fragments containing target genes were constructed. A nucleotide sequence for the Notchl receptor gene was obtained from Genebank, and the Notch1 receptor protein was analyzed using the Swiss-Prot database, to determine the extracellular structure domain, transmembrane segment and intracellular domain. The primers (5'-CCTGAGGGCTTCAAAGTGTC-3',


Figure 3. Comparison of (A) cell proliferation ability and (B) cell migration ability. AdBMP9, bone morphogenetic protein 9 adenovirus; AdR-dnNotch1, adenovirus expressing the dominant-negative mutant of Notch1.

5'-CGGAACTTCTTGGTCTCCAG-3'), which only amplified the fragments containing the extracellular structure domain and transmembrane segment of Notch1 receptor, were designed using Primer3 software (PrimerPremier \& Oligo, Whitehead Institute, Cambridge, CA, USA). The coding regions containing the extracellular domain and transmembrane segment of Notch1 receptor were used for high fidelity PCR amplification. PCR products were purified and cloned into plasmid vector pAdTrace-TOX4. The cloning steps were as follows: PCR product and vector pAdTrace-TOX4 were digested with corresponding restriction enzyme. Digested fragments were purified and ligated with T4 ligase to the pAdTrace-TOX4 plasmid. Then, the product was diluted to $200 \mu \mathrm{l}$ with $\mathrm{ddH}_{2} \mathrm{O}$ and mixed with $700 \mu \mathrm{l}$ ethanol, and centrifuged for $5 \mathrm{~min}$ at $13,000 \mathrm{x} \mathrm{g}$ and ethanol precipitation was conducted. The precipitate was subsequently dissolved

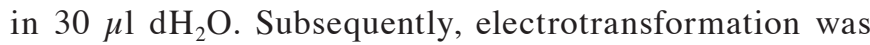
conducted followed by the selection of positive clones and identification.

Detection methods. The cell proliferation ability was detected by cresyl violet-stained (Beyotime, Shanghai, China) and quantitative analysis. Cell migration ability was detected using the scratch test. Cell cycle transition was tested using flow cytometry (BD-LSR II, BD Biosciences, San Jose, CA, USA). The expression of receptors and Notch ligands induced by BMP9 and the nucleocytoplasmic traffic level of Notch intracellular domain (NICD) were examined using RT-PCR and immunofluorescence staining. 
AdBMP9+AdR -dnNotch1
AdBMP9 +Compound E
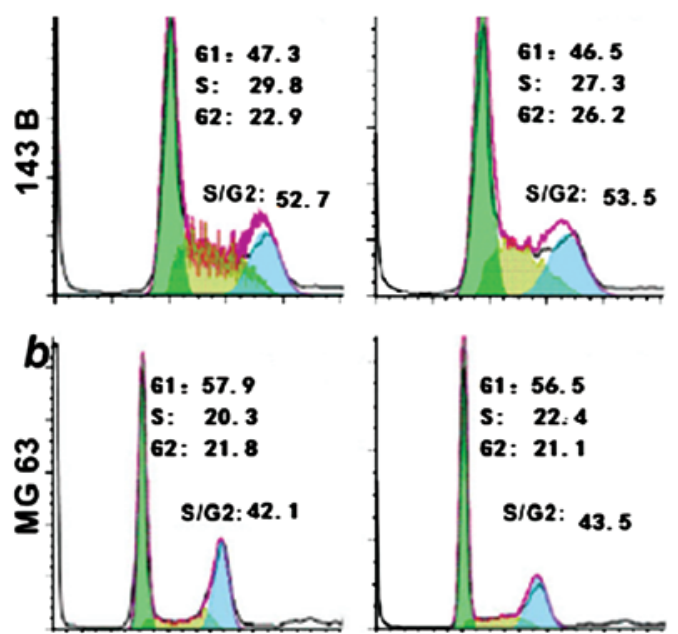

AdBMP9

AdR -dnNotch1
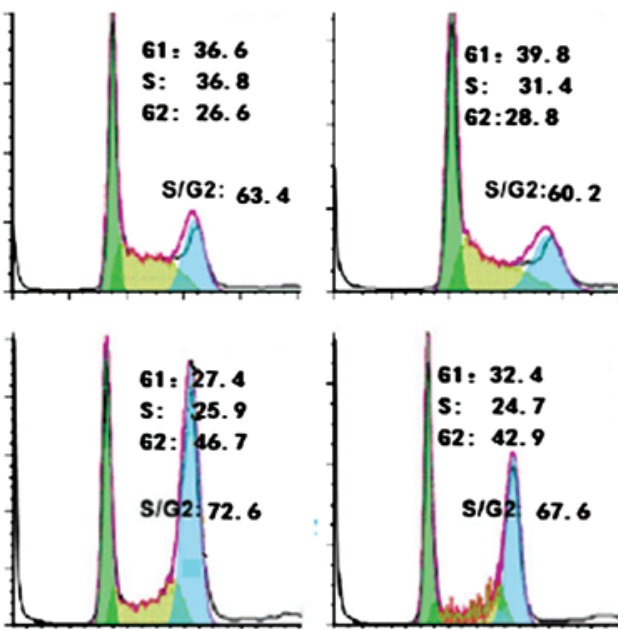

Figure 4. Comparison of the cell cycle ratio. AdBMP9, bone morphogenetic protein 9 adenovirus; AdR-dnNotch1, adenovirus expressing the dominant-negative mutant of Notch1.

Statistical analysis. SPSS 19.0 (SPSS, Inc., Chicago, IL, USA) was used for the statistical analysis. Measurement data were presented by mean \pm standard deviation. ANOVA was used for comparisons between groups. Counting data were presented as (\%), and $\chi^{2}$ test was used for comparisons among groups. $\mathrm{P}<0.05$ was considered to indicate a statistically significant difference.

\section{Results}

Ligand and Notch receptor expression. We detected high levels of expression for Notch ligands DLL1, JAG1 and JAG2, as well as Notch receptors Notch1, Notch2 and Notch3 in the two cell lines. NICD1, an intracellular reactive molecule released by Notch, which was expressed in the cells, appeared brown and uniformly distributed, suggesting a positive expression of Notch1 receptors in situ in osteosarcomas (Figs. 1 and 2).

Comparison of the ability of cell proliferation and migration. Cell proliferation ability and migration increased in the AdBMP9 group and this increase was more prominent than that in the AdBMP9+AdR-dnNotch1 and AdBMP9+compound E groups (Fig. 3). The differences were statistically significant $(\mathrm{P}<0.05)$. However, the ability of cell proliferation and migration in the AdR-dnNothcl group was lower than that in the AdBMP9 group. Differences had not statistically significant $(\mathrm{P}>0.05)$.

Comparison of the cell cycle ratio. The cell cycle ratio in the S/G2 phase increased considerably in the AdBMP9 group and the increase was more intense than that in the AdBMP9+AdR-dnNotch1 and AdBMP9+compound E groups (Fig. 4). The differences were statistically significant $(\mathrm{P}<0.05)$. Nevertheless, the cell cycle ratio in the $\mathrm{S} / \mathrm{G} 2$ phase in the AdR-dnNotch1 group was lower than that in the AdBMP9 group, although the differences were not statistically significant $(\mathrm{P}>0.05)$.

\section{Discussion}

Notch signaling is an unusual signaling pathway, whose activity does not depend on secondary messengers for amplification. Instead, Notch signaling is modulated by glycosylation, differential intracellular trafficking, and ubiquitin-dependent degradation. The Notch signaling pathway consists of various Notch receptors including Notch1, Notch2, Notch3 and Notch4; Notch ligands including Jag1, Jag2, Dll1, DIl3 and Dll4; positive and negative regulators and transcription factors (10). The majority of Notch ligands are type I transmembrane proteins. Some signaling pathways such as the transforming growth factor (TGF)- $\beta /$ Smad signaling pathway, mitogen-activated protein kinase (MAPK)-mediated signaling pathway, Wnt signaling pathway and Notch signaling pathway are involved in the regulation of the mechanism of osteogenesis and osteosarcoma process in the bone marrow mesenchymal stem cells $(11,12)$. Previous studies have demonstrated that early target gene regulation in BMP9 such as in Heyl gene was among the characteristic targets for the Notch pathway (13). Knockout mice with Notch gene showed severe skeletal abnormalities. The Dll3 mutation caused spinal ribs hypoplasia. The overexpression of Notch ligands and Notch receptors damaged the differentiation of osteoblasts and osteoclasts of progenitor cells.

The Notch receptors are composed of the extracellular, transmembrane and intracellular domains. The extracellular domain has several epidermal growth factor receptor (EGFR) (EGF-like repeats) and three Lin/Notch repeats. The intracellular domain of Notch, containing the RAM (recombination binding protein-J associated molecular) structural domain, Notch cytokine response region, six Ankyrin repeats (also known as CDC10), and proline-glutamate-serine-threonine rich region, is responsible for transferring extracellular signals to the nucleus $(14,15)$.

With the Notch ligands binding to Notch receptors, the receptor conformation is modified and the S2 and S3 sites 
within the extracellular domain are cut by a disintegrin and metalloprotease 10 and $\gamma$-secretase (2). NICD is then released and transported into the nucleus and interacts with transcription factors such as CSL/RBPJk and the mastermind-like (MAML) protein, replacing the co-repressor complex to activate CSL and collect co-activators, such as MAML and p300, and subsequently initiating transcription from the downstream target genes (2-4). The main target genes for Notch are hairy/enhancer of split (HES) family, and HES-relate repressor. Other factors involved in Notch regulation include cell cycle regulatory factors, such as p21, cyclin D1, c-Myc, nuclear factor- $\mathrm{\kappa B} 2$ and factors regulating apoptosis $(16,17)$.

The Notch proteins were shown to fulfill various functions in tumor development and the expression patterns for Notch ligands and receptors were different in the same type of tumors $(18,19)$. The Notch signaling pathway is dysregulated in many human malignancies (12). Notch1 is a typical cancer gene and carcinogenesis may be achieved by interacting with other signaling pathways, including the Ras/MAPK, TGF- $\beta$, vascular endothelial growth factor, human epidermal growth factor 2 signaling pathways (20). The results of the present study show that the ability of cell proliferation and migration in the AdBMP9 group markedly increased and this increase was more intense compared to that observed in the AdBMP9+AdR-dnNotch1 and AdBMP9+compound E groups. Nevertheless, the ability of cell proliferation and migration in the AdR-dnNothcl group was lower than that in the AdBMP9 group, although the differences were not statistically significant $(\mathrm{P}>0.05)$. The cell cycle ratio in the $\mathrm{S} / \mathrm{G} 2$ phase increased significantly in the AdBMP9 group and was higher than that in the AdBMP9+AdR-dnNotch1 and AdBMP9+compound E groups. Thus, the Notch signaling pathway potentially plays an important role in mediating the growth of osteosarcoma promoted by BMP9.

\section{References}

1. Tang N, Song WX, Luo J, Haydon RC and He TC: Osteosarcoma development and stem cell differentiation. Clin Orthop Relat Res 466: 2114-2130, 2008.

2. Sharff KA, Song WX, Luo X, Tang N, Luo J, Chen J, Bi Y, He BC, Huang J, Li X, et al: Heyl basic helix-loop-helix protein plays an important role in mediating BMP9-induced osteogenic differentiation of mesenchymal progenitor cells. J Biol Chem 284 649-659, 2009.
3. Andersson ER, Sandberg R and Lendahl U: Notch signaling: Simplicity in design, versatility in function. Development 138: 3593-3612, 2011

4. Louvi A and Artavanis-Tsakonas S: Notch and disease: a growing field. Semin Cell Dev Biol 23: 473-480, 2012.

5. Sharff KA, Song WX and Luo X: Hey 1 basic helix-loop-helix protein plays an important role in mediating BMP9-induced differentiation of mesenchynal progenitor cells. J Biol Chem 284: 649-659, 2009.

6. Kadesch T: Notch signaling the demise of elegant simplicity. Curr Opin Genet Dev 14: 506-512, 2004.

7. Walsh DW, Roxburgh SA and McGettigan P: Co-regulation of Gremlin and Notch signaling in diabetic nephropathy.Biochin Biophys Acta 1782: 10-21, 2008

8. Tao J, Chen S and Lee B: Alteration of Notch signaling in skeletal development and disease. Ann N Y Acad Sci 1192: 257-268, 2010.

9. Penton AL, Leonard LD and Spinner NB: Notch signaling in human development and disease. Semin Cell Dev Biol 23: 450-457, 2012.

10. Bolós V, Grego-Bessa J and de la Pompa JL: Notch signaling in development and cancer. Endocr Rev 28: 339-363, 2007.

11. Engin F, Bertin T, Ma O, Jiang MM, Wang L, Sutton RE, Donehower LA and Lee B: Notch signaling contributes to the pathogenesis of human osteosarcomas. Hum Mol Genet 18: 1464-1470, 2009.

12. Tanaka M, Setoguchi T, Hirotsu M, Gao H, Sasaki H, Matsunoshita Y and Komiya S: Inhibition of Notch pathway prevents osteosarcoma growth by cell cycle regulation. Br J Cancer 100: 1957-1965, 2009.

13. Zhang P, Yang Y, Nolo R, Zweidler-McKay PA and Hughes DP: Regulation of NOTCH signaling by reciprocal inhibition of HES1 and Deltex 1 and its role in osteosarcoma invasiveness. Oncogene 29: 2916-2926, 2010.

14. Zhang P, Yang Y, Zweidler-McKay PA and Hughes DP: Critical role of notch signaling in osteosarcoma invasion and metastasis. Clin Cancer Res 14: 2962-2969, 2008.

15. Li B: Bone morphogenetic protein-Smad pathway as drug targets for osteoporosis and cancer therapy. Endocr Metab Immune Disord Drug Targets 8: 208-219, 2008

16. Kubota T, Michigami T and Ozono K: Wnt signaling in bone metabolism. J Bone Miner Metab 27: 265-271, 2009.

17. Guruharsha KG, Kankel MW and Artavanis-Tsakonas S: The Notch signalling system: recent insights into the complexity of a conserved pathway. Nat Rev Genet 13: 654-666, 2012.

18. Tien AC, Rajan A and Bellen HJ: A Notch updated. J Cell Biol 184: 621-629, 2009.

19. Yin L, Velazquez OC and Liu ZJ: Notch signaling: Emerging molecular targets for cancer therapy. Biochem Pharmacol 80: 690-701, 2010.

20. Roti G, Carlton A, Ross KN, Markstein M, Pajcini K, Su AH, Perrimon N, Pear WS, Kung AL, Blacklow SC, et al: Complementary genomic screens identify SERCA as a therapeutic target in NOTCH1 mutated cancer. Cancer Cell 23: 390-405, 2013 